IJMMS 2003:4, 209-228

PII. S0161171203107089

http://ijmms.hindawi.com

(c) Hindawi Publishing Corp.

\title{
EXISTENCE OF PERIODIC SOLUTIONS AND HOMOCLINIC ORBITS FOR THIRD-ORDER NONLINEAR DIFFERENTIAL EQUATIONS
}

\author{
O. RABIEI MOTLAGH and Z. AFSHARNEZHAD
}

Received 23 July 2001 and in revised form 18 February 2002

\begin{abstract}
The existence of periodic solutions for the third-order differential equation $\dot{\dot{x}}+$ $\omega^{2} \dot{x}=\mu F(x, \dot{x}, \ddot{x})$ is studied. We give some conditions for this equation in order to reduce it to a second-order nonlinear differential equation. We show that the existence of periodic solutions for the second-order equation implies the existence of periodic solutions for the above equation. Then we use the Hopf bifurcation theorem for the second-order equation and obtain many periodic solutions for it. Also we show that the above equation has many homoclinic solutions if $F(x, \dot{x}, \ddot{x})$ has a quadratic form. Finally, we compare our result to that of Mehri and Niksirat (2001).
\end{abstract}

2000 Mathematics Subject Classification: 65Lxx.

1. Introduction. There are many papers for the existence of periodic solutions for nonlinear second-order differential equations (e.g., see [1, 2, 5]) but there are few papers for nonlinear third-order differential equations. These kind of equations arise in engineering, for example, in problems related to energy and acceleration. Because of the topological characteristics of the threedimensional space, the investigation of periodic solutions for the nonlinear third-order differential equations is a difficult problem.

In [6], Mehri and Niksirat considered the nonlinear equation

$$
\dot{\dot{X}}=F(X, \dot{X}, \ddot{X}), \quad X \in \mathbb{R}^{2 n+1}
$$

and showed that, under some conditions, there exists $\omega>0$ such that $X(\omega)=$ $X(0)$. Then, Mehri and Niksirat [7] considered the nonlinear third-order differential equation

$$
\dot{\dot{x}}+\omega^{2} \dot{x}=\mu F(x, \dot{x}, \ddot{x})
$$

and obtained some conditions for the existence of a periodic for it. We will write their result in the following form: consider (1.2), where $F$ is assumed to be smooth enough such that the existence and uniqueness of the solution are 
guaranteed. Let $\lambda=2 \pi \omega^{-1}+\mu \tau$ and define

$$
\Gamma_{\lambda}(a, b, \tau)=\left(\begin{array}{c}
x(0)-x(\lambda) \\
\dot{x}(0)-\dot{x}(\lambda) \\
\ddot{x}(0)-\ddot{x}(\lambda)
\end{array}\right) .
$$

Assume that there exist $a_{0}, b_{0} \neq 0$ such that

$$
\begin{array}{r}
\int_{0}^{2 \pi \omega^{-1}} F\left(a_{0}+b_{0} \cos \omega t,-b_{0} \sin \omega t,-b_{0} \cos \omega t\right) d t=0, \\
\int_{0}^{2 \pi \omega^{-1}} F\left(a_{0}+b_{0} \cos \omega t,-b_{0} \sin \omega t,-b_{0} \cos \omega t\right) \cos \omega t d t=0, \\
\int_{0}^{2 \pi \omega^{-1}} \int_{0}^{2 \pi \omega^{-1}}\left[h(t) F_{x}(u)-F_{x}(t) h(u)\right] \cos \omega t d t d u \neq 0,
\end{array}
$$

where

$$
\begin{gathered}
F_{v}(s)=\frac{\partial F}{\partial v}\left(a_{0}+b_{0} \cos \omega s,-b_{0} \sin \omega s,-b_{0} \cos \omega s\right), \quad v \in\{x, \dot{x}, \ddot{x}\}, \\
h(s)=\cos \omega s F_{x}(s)-\sin \omega s F_{\dot{x}}(s)-\cos \omega s F_{\ddot{x}}(s) .
\end{gathered}
$$

Then there exists $\mu_{0}>0$ such that for all $|\mu|<\mu_{0}$, (1.2) has a nonconstant $\lambda$-periodic solution. Also, they proved that if all the stationary points of (1.2) are nonperiodic and $\Gamma_{0}(a, b, \tau)$ has odd signed periodic orbits, then (1.2) has at least one signed periodic solution for $0 \leq \mu \leq 1$.

In what follows, we consider the third-order differential equation (1.2) and obtain some conditions for the existence of periodic solutions for it. These conditions are applicable to many third-order differential equations. Let $f(x, \dot{x})$ be $C^{r}(r \geq 3)$ and

$$
F(x, \dot{x}, \ddot{x})=f_{x}(x, \dot{x}) \dot{x}+f_{\dot{x}}(x, \dot{x}) \ddot{x} .
$$

We show that if $f_{x \dot{x}}(0,0) \neq 0$, then (1.2) has many periodic solutions. The conditions for the existence of the homoclinic orbits are also studied. For example, if $f(x, \dot{x})$ consists of some quadratic terms, then (1.2) has many homoclinic orbits which are filled up with periodic solutions. In Section 2, we present some mathematical preliminaries and show that the existence of periodic solutions for the second-order differential equation implies the existence of periodic solutions for the third-order differential equation. The existence of the fixed points for the second-order differential equation is discussed in Section 3. Section 4 is devoted to the existence of periodic solutions for the second-order differential equation. In Section 5, we give a few examples, and finally, we compare our results to that of [7]. 
2. Preliminaries. Our main tool in the development of this paper is the Poincaré-Andronove-Hopf's bifurcation theorem which is usually called Hopf bifurcation theorem. There are several versions of this theorem but we give the version mentioned in [4]; the interested reader can find other versions of this theorem in $[3,8]$.

THeOrem 2.1 (Poincaré-Andronove-Hopf). Let $\dot{X}=A(\lambda) X+F(X, \lambda)$ be a $C^{r}$ with $r \geq 3$, planar vector field depending on a scalar parameter $\lambda$ such that $F(0, \lambda)=0$ and $D_{X} F(0, \lambda)=0$ for all sufficiently small $|\lambda|$. Assume that the linear part $A(\lambda)$ at the origin has the eigenvalues $\alpha(\lambda) \pm i \beta(\lambda)$ with $\alpha(0)=0$ and $\beta(0) \neq 0$. Furthermore, suppose that the eigenvalues cross the imaginary axis with nonzero speed, that is, $\alpha^{\prime}(0) \neq 0$. Then, in any neighborhood $U$ of the origin in $\mathbb{R}^{2}$ and for any given $\lambda_{0}>0$, there is a $\bar{\lambda}$ with $|\bar{\lambda}|<\lambda_{0}$ such that $\dot{X}=A(\bar{\lambda}) X+F(x, \bar{\lambda})$ has a nontrivial periodic orbit in $U$.

We rewrite this theorem by the following proposition.

Proposition 2.2. Consider the $C^{r}(r \geq 3)$ planar vector field

$$
\dot{X}=F(X, \lambda), \quad \lambda \in \mathbb{R}
$$

Assume that there exists an open interval I and a $C^{1}$ map $x: I \rightarrow \mathbb{R}$ such that $F(x(\lambda), \lambda)=0$. Also, assume that the linear part of (2.1) has the eigenvalues $\alpha(\lambda) \pm i \beta(\lambda)$ with $\alpha\left(\lambda_{0}\right)=0$ and $\beta\left(\lambda_{0}\right) \neq 0$. If $\alpha^{\prime}\left(\lambda_{0}\right) \neq 0$, then in any given neighborhood $U$ of $x\left(\lambda_{0}\right)$ and for any given $\varepsilon>0$, there exists $\bar{\lambda} \in I$ with $\mid \bar{\lambda}-$ $\lambda_{0} \mid<\varepsilon$ such that $\dot{X}=F(X, \bar{\lambda})$ has a nontrivial periodic orbit in $U$.

Proof. Without loss of generality, we can assume that $\left(x\left(\lambda_{0}\right), \lambda_{0}\right)=(0,0)$. Let $\lambda \in I$, by the Taylor expansion of $F(X, \lambda)$, we have

$$
\dot{X}=D_{X} F(x(\lambda), \lambda)(X-x(\lambda))+G(X, \lambda),
$$

where $G(x(\lambda), \lambda)=0$ and $D_{X} G(x(\lambda), \lambda)=0$. If we put $D_{X} F(x(\lambda), \lambda)=A(\lambda)$, $Y=X-x(\lambda)$, and $H(Y, \lambda)=G(Y+x(\lambda), \lambda)$, then we have

$$
\dot{Y}=A(\lambda) Y+H(Y, \lambda) \text {. }
$$

Equation (2.3) is a $C^{r}$ vector field satisfying the conditions of the Hopf bifurcation theorem.

Definition 2.3. Consider (2.1) and suppose that Proposition 2.2 (Hopf bifurcation theorem) holds for it. We say that for $\lambda=\lambda_{0}$, a Hopf bifurcation occurs at $x\left(\lambda_{0}\right)$ for (2.1). 
Now we reduce the third-order differential equation to a second-order differential equation. We have

$$
\begin{gathered}
\dot{x}=y, \\
\dot{y}=z, \\
\dot{z}=-\omega^{2} y+\mu F(x, y, z) .
\end{gathered}
$$

We consider (1.2) where $F: \mathbb{R}^{3} \rightarrow \mathbb{R}$ is a $C^{r}(r \geq 2)$ map such that $F(0,0,0)=0$ and $D F(0,0,0)=0$. Suppose that there is a $C^{r+1}$ map $f: \mathbb{R}^{2} \rightarrow \mathbb{R}$ such that

$$
F(x, \dot{x}, \ddot{x})=f_{x}(x, \dot{x}) \dot{x}+f_{\dot{x}}(x, \dot{x}) \ddot{x} .
$$

Substituting (2.5) in (1.2), we obtain

$$
\frac{d}{d t}\left(\ddot{x}+\omega^{2} x-\mu f(x, \dot{x})\right)=\dot{x}+\omega^{2} \dot{x}-\mu(\underbrace{f_{x}(x, \dot{x}) \dot{x}+f_{\dot{x}}(x, \dot{x}) \ddot{x}}_{F(x, \dot{x}, \ddot{x})})=0 .
$$

Therefore,

$$
\ddot{x}+\omega^{2} x-\mu f(x, \dot{x})=k,
$$

where $k$ is a real constant. If we put $k=\omega^{2} \lambda$ and $\bar{x}=x-\lambda$, then we get

$$
\ddot{\bar{x}}+\omega^{2} \bar{x}=\mu f(\bar{x}+\lambda, \dot{\bar{x}}) .
$$

Dropping the bars, we obtain

$$
\begin{gathered}
\dot{x}=y, \\
\dot{y}=-\omega^{2} x+\mu f(x+\lambda, y) .
\end{gathered}
$$

LEMMA 2.4. The curve $(x(t), \dot{x}(t))$ is a solution of (2.9) if and only if $(x(t)+$ $\lambda, \dot{x}(t), \ddot{x}(t))$ is a solution of (2.4). Furthermore, if $(x(t), \dot{x}(t))$ is a periodic (homoclinic) solution, then $(x(t)+\lambda, \dot{x}(t), \ddot{x}(t))$ is a periodic (homoclinic) solution.

Proof. It is obvious that if $(x(t), \dot{x}(t))$ is a solution for (2.9), then $(x(t)+$ $\lambda, \dot{x}(t), \ddot{x}(t))$ is a solution for (2.4). Let $(x(t), \dot{x}(t))$ be a periodic solution with period $T$. For each $t \in \mathbb{R}$, we have

$$
\begin{aligned}
\ddot{x}(t+T) & =\lim _{h \rightarrow h} \frac{\dot{x}(t+T+h)-\dot{x}(t+T)}{h} \\
& =\lim _{h \rightarrow h} \frac{\dot{x}(t+h)-\dot{x}(t)}{h} \\
& =\ddot{x}(t) .
\end{aligned}
$$


Therefore, $(x(t)+\lambda, \dot{x}(t), \ddot{x}(t))$ is a periodic solution with period $T$. If $(x(t), \dot{x}(t))$ is a homoclinic solution with $\lim _{t \rightarrow \pm \infty}(x(t), \dot{x}(t))=\left(x_{0}, 0\right)$, then

$$
\lim _{t \rightarrow \pm \infty} \ddot{x}(t)=-\omega^{2} x_{0}+\mu f\left(x_{0}+\lambda, 0\right)=0 .
$$

Since $\left(x_{0}, 0\right)$ is a fixed point for (2.9), then $\left(x_{0}+\lambda, 0,0\right)$ is a fixed point for (2.4). Hence, $(x(t)+\lambda, \dot{x}(t), \ddot{x}(t))$ is a homoclinic solution.

REMARK 2.5. Since $F(0,0,0)=0$ and $D F(0,0,0)=0$ and since

$$
D F=\left(f_{x x} y+f_{x y} z, f_{x y} y+f_{x}+f_{y y} z, f_{y}\right),
$$

then we will have $D F(0,0,0)=\left(0, f_{x}(0,0), f_{y}(0,0)\right)=0$. Also, we can assume that $f(0,0)=0$; otherwise, we put $g(x, y)=f(x, y)-f(0,0)$. The map $g(x, y)$ satisfies (2.5). Moreover, $g(0,0)=0$ and $D g(0,0)=0$.

3. Fixed points. If $\lambda=0$, then the origin is a fixed point for (2.9). If $\lambda \neq 0$, then it is important to know fixed points of (2.9).

THEOREM 3.1. For each $0<M \in \mathbb{R}$, there exists an open rectangle $\mathscr{R} \subset \mathbb{R}^{2}$ containing $[-M, M] \times\{0\}$ and $a C^{1}$ map $x: \mathscr{R} \rightarrow \mathbb{R}$ such that $(x(\mu, \lambda), 0)$ is a fixed point for (2.9). Furthermore, the real part of the eigenvalues of the linear part of (2.9) is $\mu f_{y}(x(\mu, \lambda)+\lambda, 0)$, also for each $(\mu, 0),(0, \lambda) \in \mathscr{R}, x(\mu, 0)=x(0, \lambda)=0$.

Proof. Consider the map

$$
\begin{gathered}
B: \mathbb{R}^{3} \longrightarrow \mathbb{R} \\
(x, \mu, \lambda) \longrightarrow-\omega^{2} x+\mu f(x+\lambda, 0) .
\end{gathered}
$$

Since for each $\mu \in \mathbb{R}, B(0, \mu, 0)=0$, and $B_{x}(0, \mu, 0)=-\omega^{2} \neq 0$, then, by the implicit function theorem, there exist an open neighborhood $U_{\mu}$ containing $(\mu, 0)$ and a $C^{1}$ map $x_{\mu}: U_{\mu} \rightarrow \mathbb{R}$ such that for each $(\nu, \lambda) \in U_{\mu}, B\left(x_{\mu}(\nu, \lambda)\right)=0$. Therefore, $\left(x_{\mu}(\nu, \lambda), 0\right)$ is a fixed point for (2.9). Since $[-M, M] \times\{0\}$ is compact, so we can find $-M \leq \mu_{0}, \ldots, \mu_{k} \leq M$ such that $[-M, M] \times\{0\} \subset \bigcup_{i=0}^{k} U_{\mu_{i}}$. Let $\mathscr{R}$ be an open rectangle with $[-M, M] \times\{0\} \subset \mathscr{R} \subset \bigcup_{i=0}^{k} U_{\mu_{i}}$. If $(\nu, \lambda) \in U_{\mu_{i}} \cup U_{\mu_{j}}$, $(i \neq j)$, then, by the uniqueness of the solution for the implicit function theorem, $x_{\mu_{i}}(\nu, \lambda)=x_{\mu_{j}}(\nu, \lambda)$. This shows that we can define the $C^{1}$ map

$$
\begin{gathered}
x: \mathscr{R} \longrightarrow \mathbb{R}, \\
(\mu, \lambda) \longmapsto x_{\mu_{i}}(\mu, \lambda) \quad(\mu, \lambda) \in U_{\mu_{i}} .
\end{gathered}
$$

The linear part of $(2.9)$ at $(x(\mu, \lambda), 0)$ is

$$
A(\mu, \lambda)=\left(\begin{array}{cc}
0 & 1 \\
-\omega^{2}+\mu f_{x} & \mu f_{y}
\end{array}\right)
$$


where all partial derivatives are computed at $(x(\mu, \lambda)+\lambda, 0)$. The eigenvalues of $A(\mu, \lambda)$ are

$$
\Gamma_{1,2}=\Gamma_{1,2}(\mu, \lambda)=\frac{\mu f_{y} \pm \sqrt{\left(\mu f_{y}\right)^{2}+4\left(\mu f_{x}-\omega^{2}\right)}}{2} .
$$

If $\left(\mu f_{y}\right)^{2}+4\left(\mu f_{x}-\omega^{2}\right)<0$, then the real part of $\Gamma_{1,2}$ is $\mu f_{y}$. By Remark 2.5, $D f(0,0)=0$, so for all $\mu \in[-M, M]$, we have

$$
\lim _{\lambda \rightarrow 0}\left(\mu f_{y}\right)^{2}+4\left(\mu f_{x}-\omega^{2}\right)<0
$$

Hence, we can shrink $\mathscr{R}$ such that $[-M, M] \times\{0\} \subset \mathscr{R}$ and $\left(\mu f_{y}\right)^{2}+4\left(\mu f_{x}-\right.$ $\left.\omega^{2}\right)<0$.

LEMmA 3.2. Suppose that $\mathscr{R}$ is as in Theorem 3.1 and $(\mu, \lambda) \in \mathscr{R}$. Then the fixed point $(x(\mu, \lambda), 0)$ is isolated.

Proof. Consider the map

$$
\begin{gathered}
\Omega: \mathbb{R} \longrightarrow \mathbb{R}, \\
x \longmapsto-\omega^{2} x+\mu f(x+\lambda, 0) .
\end{gathered}
$$

We have $\Omega(x(\mu, \lambda))=0$ and $\Omega_{x}(x(\mu, \lambda))=\mu f_{x}-\omega^{2}<0$. Therefore, $\Omega$ is decreasing in a neighborhood of $x(\mu, \lambda)$; hence, $(x(\mu, \lambda), 0)$ is isolated.

REMARK 3.3. Assume that we have the system

$$
\begin{gathered}
\dot{x}=y, \\
\dot{y}=g(x, y),
\end{gathered}
$$

where $g$ is a $C^{1}$ function. Let $\gamma=(x(t), y(t))$ be a solution of this system and let $I=(a, b)$ be the interval of $y(t)>0(y(t)<0)$. If $t \in I$, then $x(t)$ is increasing (decreasing), so we can consider $y(t)$ as a $C^{1}$ function of $x(t)$. Indeed, if we define the map $Y:(x(a), x(b)) \rightarrow \mathbb{R}$ with $Y(x(t))=y(t)(t \in I)$, then $Y(t)$ is a well-defined $C^{1}$ map satisfying $(x, Y(x)) \in\{\gamma(t): t \in I\}$.

4. Periodic solutions. In this section, we obtain some conditions for (2.9) in order to have periodic solutions. We consider the set $\mathscr{R}$ and the map $x: \mathscr{R} \rightarrow \mathbb{R}$ defined in Section 3.

LEMMA 4.1. Let $(\mu, \lambda) \in \mathscr{R}$. Then $(\partial x / \partial \lambda)(\mu, \lambda) \neq-1$.

Proof. We have

$$
-\omega^{2} x(\mu, \lambda)+\mu f(x(\mu, \lambda)+\lambda, 0)=0, \quad(\mu, \lambda) \in \mathscr{R} .
$$


Computing the derivative with respect to $\lambda$, we get

$$
-\omega^{2} \frac{\partial x}{\partial \lambda}(\mu, \lambda)+\mu \frac{\partial f}{\partial x}(x(\mu, \lambda)+\lambda, 0)\left(\frac{\partial x}{\partial \lambda}(\mu, \lambda)+1\right)=0 .
$$

If $(\partial x / \partial \lambda)(\mu, \lambda)=-1$, then $-\omega^{2}(\partial x / \partial \lambda)(\mu, \lambda)=0$. This is a contradiction, so $(\partial x / \partial \lambda)(\mu, \lambda) \neq-1$.

THEOREM 4.2. Let $\left(\mu_{0}, \lambda_{0}\right) \in \mathscr{R}, \mu_{0} \neq 0$, and

$\left(\mathrm{H}_{1}\right) f_{y}\left(x\left(\mu_{0}, \lambda_{0}\right)+\lambda_{0}, 0\right)=0$,

$\left(\mathrm{H}_{2}\right) f_{x y}\left(x\left(\mu_{0}, \lambda_{0}\right)+\lambda_{0}, 0\right) \neq 0$.

Then,

(i) for $\mu=\mu_{0}$ and for $\lambda=\lambda_{0}$, a Hopf bifurcation occurs at $\left(x\left(\mu_{0}, \lambda_{0}\right), 0\right)$ for (2.9),

(ii) there exist an interval I containing $\mu_{0}$ and a $C^{1}$ map $\lambda: I \rightarrow \mathbb{R}$ such that $0 \notin I$ and $\lambda\left(\mu_{0}\right)=\lambda_{0}$. Moreover, for each $\mu \in I, \mathrm{H}_{1}$ and $\mathrm{H}_{2}$ hold for $(\mu, \lambda(\mu))$,

(iii) for each $\mu \in I$, the system

$$
\begin{gathered}
\dot{x}=y, \\
\dot{y}=z, \\
\dot{z}=-\omega^{2} y+\mu\left(f_{x}(x, y) y+f_{y}(x, y) z\right)
\end{gathered}
$$

has many periodic solutions.

Proof. (i) We have shown that the eigenvalues of the matrix $A(\mu, \lambda)$ are $\Gamma_{1,2} \cdot\left(\mathrm{H}_{1}\right)$ implies that $\operatorname{Re}\left(\Gamma_{1,2}\right)=0$. On the other hand,

$$
\frac{d \operatorname{Re}\left(\Gamma_{1,2}\right)}{d \lambda}\left(\mu_{0}, \lambda_{0}\right)=\mu_{0} f_{x y}\left(x\left(\mu_{0}, \lambda_{0}\right)+\lambda_{0}, 0\right)\left(\frac{\partial x}{\partial \lambda}\left(\mu_{0}, \lambda_{0}\right)+1\right) .
$$

By $\left(\mathrm{H}_{2}\right)$ and Lemma 4.1 , we have $\left(d \operatorname{Re}\left(\Gamma_{1,2}\right)\right) /(d \lambda)\left(\mu_{0}, \lambda_{0}\right) \neq 0$. Therefore, for $\lambda=\lambda_{0}$, a Hopf bifurcation occurs at $\left(x\left(\mu_{0}, \lambda_{0}\right), 0\right)$ for $(2.9)$.

(ii) Since $x(\mu, \lambda)$ is a $C^{1}$ map, there exists a neighborhood $W$ of $\left(\mu_{0}, \lambda_{0}\right)$ with $W \subset \mathscr{R}$, such that, for each $(\mu, \lambda) \in W$,

$$
f_{x y}(x(\mu, \lambda)+\lambda, 0)\left(\frac{\partial x}{\partial \lambda}(\mu, \lambda)+1\right) \neq 0
$$

Now, we consider the map

$$
\begin{gathered}
\Psi: W \longrightarrow \mathbb{R}, \\
(\mu, \lambda) \longmapsto f_{y}(x(\mu, \lambda)+\lambda, 0) .
\end{gathered}
$$


By $\left(\mathrm{H}_{1}\right), \Psi\left(\mu_{0}, \lambda_{0}\right)=0$. Also, we have

$$
\frac{\partial \Psi}{\partial \lambda}\left(\mu_{0}, \lambda_{0}\right)=f_{x y}\left(x\left(\mu_{0}, \lambda_{0}\right)+\lambda_{0}, 0\right)\left(\frac{\partial x}{\partial \lambda}\left(\mu_{0}, \lambda_{0}\right)+1\right) \neq 0 .
$$

By the implicit function theorem, there exists an interval $I$ containing $\mu_{0}$ and a $C^{1}$ map $\lambda: I \rightarrow \mathbb{R}$ such that $\lambda\left(\mu_{0}\right)=\lambda_{0}$. Moreover, for each $\mu \in I,(\mu, \lambda(\mu)) \in W$ and $\Psi(\mu, \lambda(\mu))=0$. We can shrink $I$ such that $0 \notin I$ and so, for each $\mu \in I,\left(\mathrm{H}_{1}\right)$, and $\left(\mathrm{H}_{2}\right)$ hold for $(\mu, \lambda(\mu))$.

(iii) Let $\mu_{1} \in I$. By (i), for $\lambda=\lambda\left(\mu_{1}\right)$, a Hopf bifurcation occurs at $\left(x\left(\mu_{1}\right.\right.$, $\left.\left.\lambda\left(\mu_{1}\right)\right), 0\right)$ for (2.9). Therefore, for each $\epsilon_{1}>0$, we can find $\bar{\lambda}_{1}$ with $\left|\bar{\lambda}_{1}-\lambda\left(\mu_{1}\right)\right|<$ $\varepsilon_{1}$, such that

$$
\begin{gathered}
\dot{x}=y, \\
\dot{y}=-\omega^{2} x+\mu_{1} f\left(x+\bar{\lambda}_{1}, y\right)
\end{gathered}
$$

has a nontrivial periodic solution $\gamma_{1}$ in the open disk $D\left(x\left(\mu_{1}, \bar{\lambda}_{1}\right), \epsilon_{1}\right)$. Consider $\epsilon_{2}>0$ such that $\gamma_{1} \cap D\left(x\left(\mu_{1}, \bar{\lambda}_{1}\right), \epsilon_{2}\right)=\phi$. We can find $\bar{\lambda}_{2}$ with $\left|\bar{\lambda}_{2}-\lambda\left(\mu_{1}\right)\right|<\varepsilon_{2}$, such that

$$
\begin{gathered}
\dot{x}=y, \\
\dot{y}=-\omega^{2} x+\mu_{1} f\left(x+\bar{\lambda}_{2}, y\right)
\end{gathered}
$$

has a nontrivial periodic solution $\gamma_{2}$ in the open disk $D\left(x\left(\mu_{1}, \bar{\lambda}_{2}\right), \epsilon_{2}\right)$. Using this method, we can find many nontrivial periodic solutions (depending on $\lambda$ ) for (2.9). Hence, by Lemma 2.4, (4.3) has many nontrivial periodic solutions.

If $\lambda_{0}=0$, then $\left(\mathrm{H}_{1}\right)$ holds automatically. Indeed $x\left(\mu_{0}, 0\right)=0$ hence, $f\left(x\left(\mu_{0}\right.\right.$, $0), 0)=0$. In this case, if

$$
f_{x y}(0,0)=f_{x y}\left(x\left(\mu_{0}, 0\right)+0,0\right) \neq 0
$$

then $\left(\mathrm{H}_{2}\right)$ holds too. Therefore, by Theorem 4.2, for $\lambda=0$, a Hopf bifurcation occurs at the origin for (2.9).

COROLlary 4.3. Let $f: \mathbb{R}^{2} \rightarrow \mathbb{R}$ be a $C^{3}$ function such that $f(0,0)=0$ and $D f(0,0)=0$. If $f_{x y}(0) \neq 0$, then, for each $(\mu, 0) \in \mathscr{R}$, the equation

$$
\dot{\ddot{x}}+\omega^{2} \dot{x}-\mu\left(\dot{x} f_{x}(x, \dot{x})+\ddot{x} f_{y}(x, \dot{x})\right)=0
$$

has many periodic solutions.

COROLlary 4.4. Let $g: \mathbb{R}^{2} \rightarrow \mathbb{R}$ be a $C^{3}$ map such that $g(0,0)=0$ and $D g(0,0)=0$. Moreover, suppose that $g_{x y}(0,0) \neq-a$ where $a \in \mathbb{R}$ is constant. 
Then, for each $(\mu, 0) \in \Re$, the equation

$$
\dot{\ddot{x}}+\omega^{2} \dot{x}-\mu\left(a \dot{x}+g_{x}(x, \dot{x})\right) \dot{x}-\mu\left(a x+g_{y}(x, \dot{x})\right) \ddot{x}=0
$$

has many periodic solutions.

Proof. Consider (2.9) with $f(x, y)=a x y+g(x, y)$. We have

$$
\begin{gathered}
\dot{x}=y, \\
\dot{y}=-\omega^{2} x+\mu(a(x+\lambda) y+g(x+\lambda, y)) .
\end{gathered}
$$

For $\lambda=0$ and each $\mu \in \mathbb{R},(0,0)$ is a fixed point for (4.13). Since

$$
\frac{\partial^{2}(a x y+g(x, y))}{\partial x \partial y}(0,0)=a+g_{x y}(0,0) \neq 0,
$$

then by Theorem 4.2 and Corollary 4.3 , for $\lambda=0$, a Hopf bifurcation occurs at the origin for (4.13). Therefore, (4.12) has many periodic solutions.

COROLlary 4.5. Let $f: \mathbb{R} \rightarrow \mathbb{R}$ be a $C^{3}$ function and $f(0)=D f(0)=0$. For each $(\mu, 0) \in \mathscr{R}$, the equations

$$
\begin{aligned}
& \dot{\dot{x}}+\omega^{2} \dot{x}-\mu\left(\dot{x}^{2}+x \ddot{x}+\ddot{x} D f(\dot{x})\right)=0, \\
& \dot{x}+\omega^{2} \dot{x}-\mu\left(\dot{x}^{2}+\dot{x} D f(x)+x \ddot{x}\right)=0
\end{aligned}
$$

have many periodic solutions.

Proof. By Theorem 4.2 and Corollary 4.3, for $\lambda=0$, a Hopf bifurcation occurs at the origin for the following equations:

$$
\begin{aligned}
& \dot{y}=-\omega^{2} x+\mu((x+\lambda) y+f(y)), \quad \dot{x}=y, \\
& \dot{y}=-\omega^{2} x+\mu((x+\lambda) y+f(x+\lambda)), \quad \dot{x}=y .
\end{aligned}
$$

Hence,

$$
\begin{aligned}
& \dot{\ddot{x}}+\omega^{2} \dot{x}-\mu\left(\dot{x}^{2}+x \ddot{x}+\ddot{x} D f(\dot{x})\right)=0, \\
& \dot{\ddot{x}}+\omega^{2} \dot{x}-\mu\left(\dot{x}^{2}+\dot{x} D f(x)+x \ddot{x}\right)=0
\end{aligned}
$$

have many periodic solutions.

THEOREM 4.6. Let $f: \mathbb{R}^{2} \rightarrow \mathbb{R}$ be a $C^{r}$ map which satisfies the condition

(H1) $f(x, y)$ is symmetric with respect to $y$-axis, that is, $x, y \in \mathbb{R}, f(x, y)=$ $f(x,-y)$. Also, let $\gamma(t)=(x(t), y(t))$ be a solution of the equation

$$
\begin{gathered}
\dot{x}=y, \\
\dot{y}=-\omega^{2} x+f(x, y) .
\end{gathered}
$$


Suppose that $\gamma$ intersects the $x$-axis at distinct points $\left(x_{1}, 0\right)$ and $\left(x_{2}, 0\right)$. Then,

(i) if both $\left(x_{1}, 0\right)$ and $\left(x_{2}, 0\right)$ are regular points, then $\gamma$ is a periodic solution;

(ii) if $\left(x_{1}, 0\right)$ is a fixed point and $\left(x_{2}, 0\right)$ is a regular point, then $\gamma$ is a homoclinic;

(iii) if both $\left(x_{1}, 0\right)$ and $\left(x_{2}, 0\right)$ are fixed points, then $\gamma$ is a part of a heteroclinic.

Proof. (i) We can assume that $\left(x_{1}, 0\right)$ and $\left(x_{2}, 0\right)$ are such that $\gamma$ does not intersect the $x$-axis between $\left(x_{1}, 0\right)$ and $\left(x_{2}, 0\right)$. We define the part of $\gamma$ which lies between $\left(x_{1}, 0\right)$ and $\left(x_{2}, 0\right)$ by

$$
\begin{gathered}
\alpha:[0, T] \longrightarrow \mathbb{R}^{2}, \\
t \longmapsto(x(t), y(t)),
\end{gathered}
$$

where $T>0, \alpha(0)=\left(x_{1}, 0\right)$ and $\alpha(T)=\left(x_{2}, 0\right)$. If we define

$$
\begin{gathered}
\beta_{1}:[-T, 0] \longrightarrow \mathbb{R}^{2}, \\
t \longmapsto(x(-t),-y(-t)),
\end{gathered}
$$

then $\beta_{1}(t)$ is a solution of the equation such that its graph is symmetric with $\alpha$, with respect to the $x$-axis. Furthermore, $\beta_{1}(0)=\left(x_{1}, 0\right)$ and $\beta_{1}(-T)=\left(x_{2}, 0\right)$. So, $\beta_{1}$ lies along $\alpha$ and hence, $\gamma$ is periodic.

(ii) Without loss of generality, we can suppose that $\gamma(0)=\left(x_{2}, 0\right)$ and $\lim _{t \rightarrow-\infty} \gamma(t)=\left(x_{1}, 0\right)$. Then the curve

$$
\begin{gathered}
\beta_{2}:[0, \infty] \longrightarrow \mathbb{R}^{2}, \\
t \longmapsto(x(-t),-y(-t))
\end{gathered}
$$

is a solution of the equation such that its graph is symmetric with $\gamma$, with respect to the $x$-axis. Furthermore, $\lim _{t \rightarrow \infty} \beta_{2}(t)=\left(x_{1}, 0\right)$ and $\beta_{2}(0)=\left(x_{2}, 0\right)$. Hence, $\beta_{2}$ lies along $\gamma$ and so $\gamma$ is a homoclinic.

(iii) The proof is similar to the proof of (ii).

5. Application. In this section, we present a few examples of the applications of Theorem 4.2 and other results of this paper. We are going to study the effect of some second-order terms on (2.4). For this purpose, we put $g(x, y, \lambda)=$ $f(x+\lambda, y)$. We know that $g(0,0,0)=0$ and $D g(0,0,0)=0$. By the Taylor expansion of $g$, we obtain

$$
\begin{aligned}
g(x, y, \lambda)= & \left(g_{x x}(0,0,0) x^{2}+g_{y y}(0,0,0) y^{2}\right. \\
& +g_{\lambda \lambda}(0,0,0) \lambda^{2}+2 g_{x y}(0,0,0) x y \\
& \left.+2 g_{x \lambda}(0,0,0) x \lambda+2 g_{y \lambda}(0,0,0) y \lambda\right)+o(3) .
\end{aligned}
$$


By definition of $g$, we have $g_{x x}(0,0,0)=g_{x \lambda}(0,0,0)=g_{\lambda \lambda}(0,0,0)=f_{x x}(0,0)$, $g_{x y}(0,0,0)=g_{y \lambda}(0,0,0)=f_{x y}(0,0)$, and $g_{y y}(0,0,0)=f_{y y}(0,0)$. Putting $f_{x x}(0,0)=a, f_{x y}(0,0)=b, f_{y y}(0,0)=c$ and truncatting the Taylor expansion at $\boldsymbol{o}(2)$, we get

$$
\begin{gathered}
\dot{x}=y, \\
\dot{y}=a \mu \lambda^{2}+\left(2 a \mu \lambda-\omega^{2}\right) x+2 b \mu \lambda y+\mu\left(a x^{2}+2 b x y+c y^{2}\right) .
\end{gathered}
$$

Hence, (5.2) can be considered as

$$
\begin{gathered}
\dot{x}=y, \\
\dot{y}=-\omega^{2} x+\mu f(x+\lambda, y),
\end{gathered}
$$

where $f(x, y)=a x^{2}+2 b x y+c y^{2}$.

If $b \neq 0$, then $f_{x y}(0,0)=b \neq 0$, hence by Corollary 4.3 , for $\lambda=0$, a Hopf bifurcation occurs for (5.2) at the origin. By Theorem 4.2, we have the following corollary.

COROLlary 5.1. If $b \neq 0$, then the equation

$$
\dot{\dot{x}}+\omega^{2} \dot{x}=\mu(a x+b \dot{x}) \dot{x}+\mu(b x+c \dot{x}) \ddot{x}
$$

has many periodic solutions.

EXAMPLE 5.2. We consider (5.2) with $a=b=0$ and $c \neq 0$. In this case, (5.2) has the following form:

$$
\begin{gathered}
\dot{x}=y, \\
\dot{y}=-\omega^{2} x+\mu c y^{2} .
\end{gathered}
$$

Putting $v=\mu c$, we obtain

$$
\begin{gathered}
\dot{x}=y, \\
\dot{y}=-\omega^{2} x+v y^{2} .
\end{gathered}
$$

We consider (5.6) where $v>0$ (the case $v<0$ is similar). The equation has $(0,0)$ as the only fixed point. On the curve $x=\left(v / \omega^{2}\right) y^{2}$, we have $\dot{y}=0$, in addition, the sign of $\dot{y}$ is shown in Figure 5.1. Therefore, a solution $\gamma=(x(t), y(t))$ of (5.6), which intersects the positive $x$-axis, is as shown in Figure 5.2. Since in region (I) of Figure 5.1, $\dot{y}<0$, so $\gamma(t)$ intersects the curve $x=\left(v / \omega^{2}\right) y^{2}$ transversally. After passing through $x=\left(v / \omega^{2}\right) y^{2}, y(t)$ increases, hence $\gamma(t)$ intersects the $x$-axis or converges to the origin. In the first case, by Theorem 4.6, $\gamma(t)$ is a periodic solution. In the second case, since $y(t)$ in region (II) of Figure 5.1 is increasing, therefore $\gamma$ cannot intersect the negative $y$-axis. On the other hand, the slope of the curve $x=\left(v / \omega^{2}\right) y^{2}$ where $x \rightarrow 0^{+}$is 


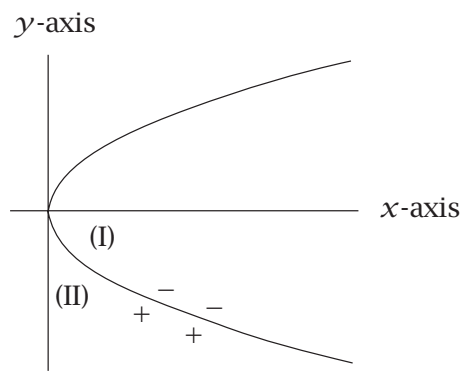

FIGURE 5.1. The sign of $\dot{y}$.

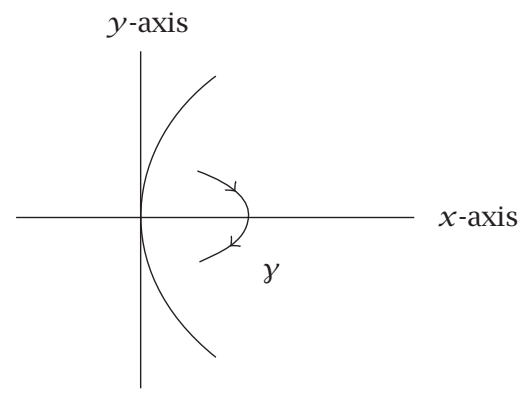

FiguRE 5.2. The local behavior of $\gamma$.

unbounded. Hence, we have

$$
\lim _{t \rightarrow \infty}\left|\frac{\dot{y}}{\dot{x}}\right|=\infty \text {. }
$$

After passing through $x=\left(\nu / \omega^{2}\right) y^{2}, \dot{y}>0$, so for sufficiently large $t$, we have $|y(t)|<1$. Hence,

$$
x(t)<\frac{v}{\omega^{2}} y^{2}(t)<\frac{v}{\omega^{2}}|y(t)|
$$

Therefore, we have

$$
\left|\frac{\dot{y}}{\dot{x}}\right|<\frac{\left|-\omega^{2} x+v y^{2}\right|}{|y|}<v+v|y(t)|
$$

which shows that

$$
\lim _{t \rightarrow \infty}\left|\frac{\dot{y}}{\dot{x}}\right| \leq v .
$$

This is a contradiction. Consequently, $\gamma$ is a periodic solution and so, $(0,0)$ is a center for (5.6). 
COROLlaRY 5.3. For each $\mu \in \mathscr{R}$, the equation

$$
\dot{\ddot{x}}+\omega^{2} \dot{x}-\mu c(\dot{x} \ddot{x})=0, \quad(c \in \mathbb{R})
$$

has many periodic solutions.

EXAMPLE 5.4. We consider (5.2) with $b=c=0$ and $a \neq 0$. In this case, (5.2) is

$$
\begin{gathered}
\dot{x}=y, \\
\dot{y}=a \mu \lambda^{2}+\left(2 a \mu \lambda-\omega^{2}\right) x+a \mu x^{2} .
\end{gathered}
$$

Setting $v=a \mu$, we get

$$
\begin{gathered}
\dot{x}=y, \\
\dot{y}=v \lambda^{2}+\left(2 v \lambda-\omega^{2}\right) x+v x^{2} .
\end{gathered}
$$

If $v \neq 0$ and $(\nu, \lambda) \in\left\{(v, \lambda): v \lambda<\omega^{2} / 4\right\}$, then (5.13) has two different fixed points $(\alpha(\nu, \lambda) \pm \beta(\nu, \lambda), 0)$ where $\alpha(\nu, \lambda)=\left(\omega^{2}-2 v \lambda\right) / 2 v$ and $\beta(\nu, \lambda)=$ $\sqrt{\omega^{4}-4 v \lambda \omega^{2}} / 2 v$. For convenience, we put $\alpha=\alpha(\nu, \lambda)$ and $\beta=\beta(\nu, \lambda)$. It can be easily checked that $(\alpha+\beta, 0)$ is a saddle point and the corresponding eigenvalues of $(\alpha-\beta, 0)$ are purely imaginary. Also (5.13) is a Hamiltonian system with Hamiltonian function

$$
H(x, y)=\frac{y^{2}}{2}-v \lambda^{2} x+\left(\omega^{2}-2 v \lambda\right) \frac{x^{2}}{2}-v \frac{x^{3}}{3}
$$

The trajectories of (5.13) are obtained by $H(x, y)=h$ where $h \in \mathbb{R}$. We put $f(x)=2 v \lambda^{2} x+\left(2 v \lambda-\omega^{2}\right) x^{2}+\left(2 v x^{3}\right) / 3$ and $g_{h}(x)=f(x)+h$. Then the trajectories of (5.13) are

$$
y^{2}=g_{h}(x), \quad(h \in \mathbb{R}), g_{h}(x) \geq 0 .
$$

Let $v \neq 0,(\nu, \lambda) \in \mathscr{R}$, and $h_{p}=-f(\alpha+\beta)$. Then $g_{h_{p}}(\alpha+\beta)=0$ and $g_{h_{p}}^{\prime}(\alpha+$ $\beta)=g_{h_{p}}^{\prime}(\alpha-\beta)=0$. Also $\alpha-\beta$ is the maximal point and $\alpha+\beta$ is the minimal point for $g_{h_{p}}(x)$. Hence, considering the graph of $g_{h_{p}}(x),(5.13)$ has a homoclinic for the saddle point $(\alpha+\beta, 0)$ (see Figures 5.3 and 5.4).

Now let $\hbar=-f(\alpha-\beta)$. For each $\delta \geq 0$, we put $h_{\delta}=\hbar+\delta$. Then $g_{h_{\delta}}(\alpha-\beta)=\delta$. On the other hand, $g_{h_{\delta}^{\prime}}^{\prime}(\alpha-\beta)=g_{h_{\delta}}^{\prime}(\alpha+\beta)=0$. Moreover, $\alpha-\beta$ is the maximal point and $\alpha+\beta$ is the minimal point for $g_{h_{\delta}}(x)$. Since $g_{h_{0}}(\alpha-\beta)=0$ and the roots of $g_{h_{\delta}}(x)=0$ varies continuously with respect to $\delta$, so for $\delta$ sufficiently small, $y^{2}=g_{h_{\delta}}(x)$ indicates a nontrivial periodic solution. Hence, $(\alpha-\beta, 0)$ is a center and the homoclinic is filled up with the periodic solutions. We apply these results to (2.4). 


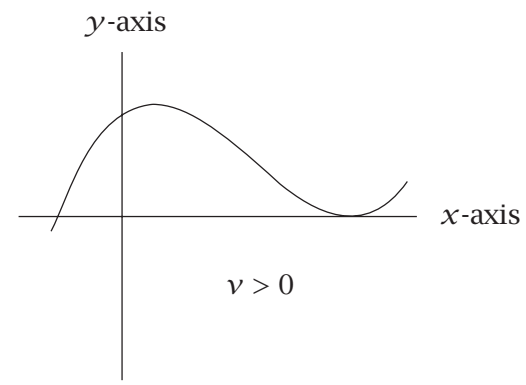

FIGURE 5.3. The graph of $g_{h}(x)$.

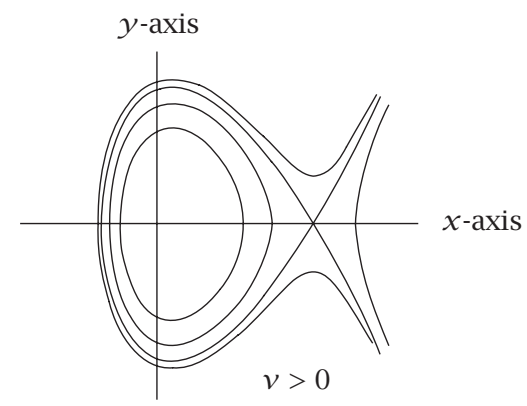

Figure 5.4. The phase space of equation (5.13).

COROLLARY 5.5. For each $\mu \in \mathscr{R}$, the equation

$$
\begin{gathered}
\dot{x}=y, \\
\dot{y}=z, \\
\dot{z}=-\omega^{2} x+a \mu x y
\end{gathered}
$$

has many homoclinic orbits and periodic solutions. The homoclinic orbits make a two-dimensional $C^{1}$ surface. In addition, each homoclinic orbit lies on a $C^{1}$ two-dimensional orientable manifold and inside it is filled up with the periodic solutions (see Figure 5.5).

Proof. By Lemma 2.4, there exist many homoclinic orbits and periodic solutions. It can be easily checked that, the graph of $g_{h_{p}}$ varies $C^{1}$ with respect to $(\nu, \lambda)$. So, by Lemma 2.4 , we conclude that the homoclinic orbits make a two-dimensional $C^{1}$ surface. Finally, since the solutions of (5.15) are distinct and varies $C^{1}$ with respect to the initial conditions, so for each $(\nu, \lambda) \in \mathscr{R}$, the solutions of (5.15) make a $C^{1}$ two-dimensional orientable manifold in the phase space of the third-order equation. 


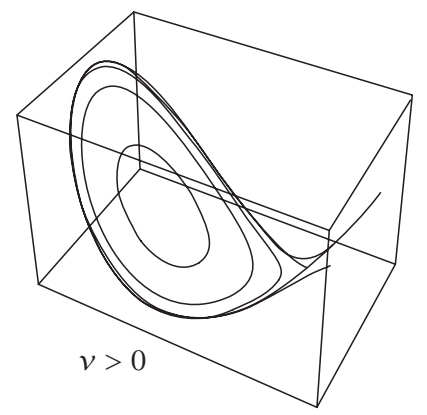

FIGURE 5.5. The two-dimensional manifold according to equation (5.13).

EXAMPLE 5.6. Consider (5.2) with $b=0$ and $a, c \neq 0$. In this case, (5.2) is reduced to

$$
\begin{gathered}
\dot{x}=y, \\
\dot{y}=a \mu \lambda^{2}+\left(2 a \mu \lambda-\omega^{2}\right) x+a \mu x^{2}+c \mu y^{2} .
\end{gathered}
$$

Setting $v=\mu a$ and $d=c / a$, we get

$$
\begin{gathered}
\dot{x}=y, \\
\dot{y}=-\omega^{2} x+v f(x+\lambda, y),
\end{gathered}
$$

where $f(x, y)=x^{2}+d y^{2}$. For each $v \neq 0$ and $(\nu, \lambda) \in\left\{(\nu, \lambda): \nu \lambda<\omega^{2} / 4\right\}$, (5.18) has two fixed points $(\alpha \pm \beta, 0)$ where $\alpha$ and $\beta$ are as in Example 5.4. It is easy to check that $(\alpha+\beta, 0)$ is a hyperbolic saddle point and the corresponding eigenvalues of $(\alpha-\beta, 0)$ are purely imaginary. Putting $\bar{x}=x-(\alpha+\beta, 0)$ and $\bar{y}=y$, we obtain

$$
\begin{gathered}
\dot{\bar{x}}=\bar{y}, \\
\dot{\bar{y}}=\sqrt{\omega^{4}-4 v \lambda \omega^{2}} \bar{x}+v \bar{x}^{2}+d v \bar{y}^{2} .
\end{gathered}
$$

For convenience, we drop the bars and obtain

$$
\begin{gathered}
\dot{x}=y, \\
\dot{y}=\sqrt{\omega^{4}-4 v \lambda \omega^{2}} x+v x^{2}+d v y^{2} .
\end{gathered}
$$

This system has two fixed points $(0,0)$ and $\left(x_{0}, 0\right)=\left(-\sqrt{\omega^{4}-4 v \lambda \omega^{2}} / \nu, 0\right)$. We consider two cases.

CASE $1(d>0)$. In this case, the local phase space of (5.20) is shown in Figure 5.6. We consider $v<0\left(v>0\right.$ is similar). Let $W^{u}(0)$ be the trajectory of a solution $\gamma(t)=(x(t), y(t))$ of (5.20). If $\gamma(t)$ is unbounded in the first region of the plan, then $\dot{y}(t) \rightarrow-\infty$. Hence, for $t>0$ sufficiently large, $y(t)$ decreases. 


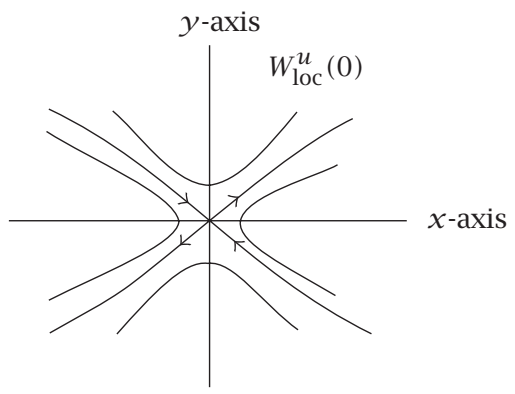

FIGURE 5.6. The local phase space of (5.18).

So, $\gamma(t)$ crosses the positive $x$-axis or converges to $\left(x_{0}, 0\right)$. In the first case, by Theorem 4.6, $\gamma(t)$ is a homoclinic. The second case shows that $\gamma(t)$ is a part of a heteroclinic. If the second case occurs, then each solution of (5.20), which lies in heteroclinic, is bounded. By the Poincaré-Bendixon theorem about two dimensional flows, the solutions (as $t \rightarrow \pm \infty$ ) tend to a cycle or a fixed point. Since each closed orbit in the plane has a fixed point in it and there is no fixed point in the inner region of the heteroclinic, so this heteroclinic is filled up with homoclinics emerged from $\left(x_{0}, 0\right)$ (see Figure 5.7). We claim that it is a contradiction. If $\gamma$ is a heteroclinic, then there exists $T>0$ such that for each $t>T, \dot{y}<0$ and so

$$
h(x)=\sqrt{-\frac{1}{v d}\left(\sqrt{\omega^{4}-4 v \lambda \omega^{2}} x+v x^{2}\right)}<y .
$$

By Remark 3.3, we can consider $y(t)$ as a function of $x(t)$. For $x(T)<x<$ $x_{0}$, we have $h(x)<y(x)$ and $\lim _{x \rightarrow x_{0}^{-}} y(x)=\lim _{x \rightarrow x_{0}^{-}} h(x)=0$. Furthermore, since $y(x)$ and $h(x)$ are decreasing, then

$$
\frac{y(x)-y\left(x_{0}\right)}{x-x_{0}}<\frac{h(x)-h\left(x_{0}\right)}{x-x_{0}} \quad\left(x(T)<x<x_{0}\right) .
$$

Hence, by the mean value theorem,

$$
\frac{d y}{d x}\left(\xi_{x}\right)<\frac{d h}{d x}\left(\eta_{x}\right) \quad\left(x<\xi_{x}, \eta_{x}<x_{0}\right)
$$

Now if $x \rightarrow x_{0}^{-}$, then $\xi_{x}, \eta_{x} \rightarrow x_{0}$. But $\lim _{x \rightarrow x_{0}^{-}} d h / d x=-\infty$, and then $\lim _{x \rightarrow x_{0}^{-}} d y / d x=-\infty$. On the other hand, from (5.20), we have

$$
\frac{d y}{d x}=\frac{\sqrt{\omega^{4}-4 v \lambda \omega^{2}} x+v x^{2}}{y}+d v y
$$




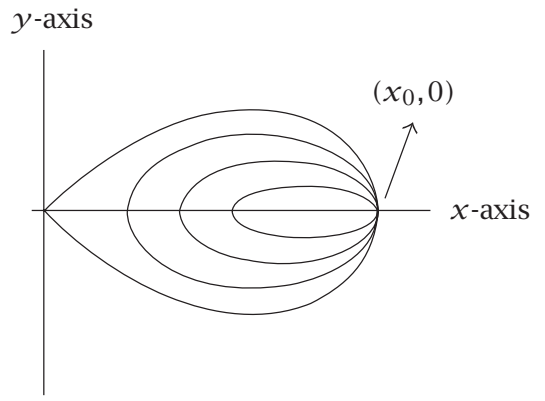

FIGURE 5.7. The heteroclinic orbit filled by homoclinic orbits.

For $x(T)<x<x_{0},\left(\sqrt{\omega^{4}-4 v \lambda \omega^{2}} x+v x^{2}\right) / y>0$ and $\lim _{x \rightarrow x_{0}^{-}} d v y(x)=0$. This result shows that $d y / d x$ does not converge to $-\infty$, which is a contradiction. Hence, $\gamma(t)$ is a homoclinic. Similarly, a solution of (5.20), which lies in the homoclinic, does not converge to $\left(x_{0}, 0\right)$, hence, it crosses $x$-axis at two different points. By Theorem 4.6, this solution is a periodic solution. Therefore, the homoclinic is filled up with the periodic solutions.

CASE $2(d<0)$. In this case, the phase space of (5.20) is as in Figure 5.6. We consider $v<0\left(v>0\right.$ is similar). For $p \in \mathbb{R}$, let $\gamma_{p}(t)=\left(x_{p}(t), y_{p}(t)\right)$ be a solution of (5.20) such that $\gamma_{p}(0)=(p, 0)$. If $0<p<x_{0}$, then $\dot{y}_{p}(0)>0$ and if $p>x_{0}$, then $\dot{y}(0)<0$. Now let $0<p<x_{0}<q$, then $\gamma_{p}(t)$ and $\gamma_{q}(t)$ are as shown in Figure 5.8. The orbits of $\gamma_{p}$ and $\gamma_{q}$ will be similar to the ones in Figures 5.8(a), 5.8(b) or 5.8(c). Figure 5.8(a) shows that $\gamma_{p}$ is a periodic solution. If Figure 5.8(b) occurs (Figure 5.8(c) is similar), then $\gamma_{p}(t)$ crosses the $x$-axis at two different points or converges to $\left(x_{0}, 0\right)$. Similar to the case $d>0$, we can show that $\gamma_{p}(t)$ does not converge to $\left(x_{0}, 0\right)$, hence by Theorem $4.6, \gamma_{p}(t)$ is a periodic solution. Also for each $p<\xi<x_{0}, \gamma_{\xi}(t)$ is a periodic solution and so $\left(x_{0}, 0\right)$ is a center.

COROLLARY 5.7. For each $\mu \in \mathscr{R}$, the system

$$
\begin{gathered}
\dot{x}=y, \\
\dot{y}=z, \\
\dot{z}=-\omega^{2} y+\mu(a x+c z) y \quad(a, c \in \mathbb{R})
\end{gathered}
$$

has many periodic solutions. In addition, if ac $>0$, then the system has many homoclinic orbits which make a $C^{1}$ two-dimensional surface. In this case, each homoclinic orbit lies on a $C^{1}$ orientable two-dimensional manifold and inside it is filled up with the periodic solutions.

Proof. By Lemma 2.4 and similar proof as Corollary 5.5, it is obvious. 


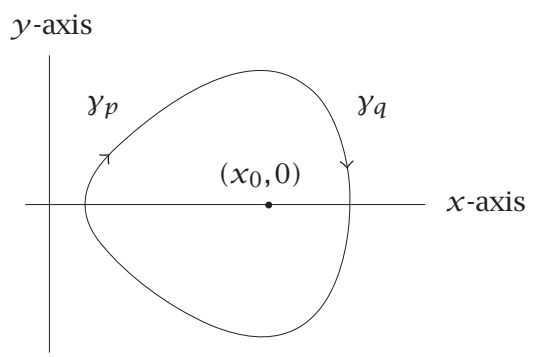

(a)

$y$-axis

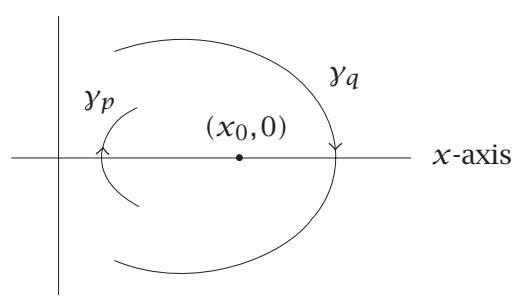

(b)

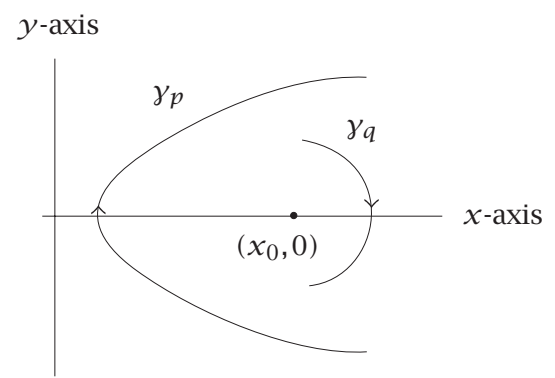

(c)

FIGURE 5.8. The local behaviour of $\gamma_{q}$ and $\gamma_{p}$.

Corollary 5.1 shows application of Theorem 4.2, where the Hopf bifurcation occurs for $\lambda=0$. Now we give an example of the application of Theorem 4.2 with $\lambda \neq 0$.

EXAMPLE 5.8. Consider (2.9) with $\mu=1, \omega=3$, and $f(x, y)=x^{2}+x^{3} y-$ $((7-3 \sqrt{5}) / 2) x^{2} y$. Then we have the following equation:

$$
\begin{gathered}
\dot{x}=y, \\
\dot{y}=\lambda^{2}-(9-2 \lambda) x+x^{2}+y\left((x+\lambda)^{3}-\frac{9-3 \sqrt{5}}{2}(x+\lambda)^{2}\right) .
\end{gathered}
$$


Since $f_{x y}(0,0)=0$, we cannot apply Corollary 4.3. But for each $\lambda$ close to 1 , we have $\left(x_{\lambda}, 0\right)=((9-2 \lambda-\sqrt{81-36 \lambda}) / 2,0)$ as a fixed point for (5.26). For $\lambda=1, x_{1}=(7-3 \sqrt{5}) / 2$ with corresponding eigenvalues $\pm i \sqrt{3 \sqrt{5}}$. Furthermore, $f_{y}\left(x_{1}+1,0\right)=0$ and $f_{x y}\left(x_{1}+1,0\right)=(9-3 \sqrt{5}) / 2 \neq 0$. Hence, for $\lambda=1$, a Hopf bifurcation occurs for $(5.26)$ at $\left(x_{1}, 0\right)$ and we have the following corollary.

COROLLARY 5.9. The following system has many periodic solutions:

$$
\dot{\ddot{x}}+\omega^{2} \dot{x}=\mu\left[\left(2 x+3 x^{2} y+(7+3 \sqrt{5}) x \dot{x}\right) \dot{x}+\left(x^{3}+\frac{7+3 \sqrt{5}}{2} x^{2}\right) \ddot{x}\right]
$$

COMPARISON. In Section 2, we explained the result of [7] briefly. Now we compare our results to that of [7].

(1) As a restriction, [7] guarantees only one periodic solution, also the value of $|\mu|$ may be very small; but by Theorem 4.2 , we obtain many periodic solutions. Moreover, we have no restriction for $|\mu|$. This is due to the fact that the constant $M$ (see Theorem 3.1) can be arbitrary large, so by Corollary 4.3, if $f_{x y}(0,0) \neq 0$, then for each $\mu \in[-M, M],(2.4)$ has many periodic solutions.

(2) It also seems that the conditions $\left(\mathrm{H}_{1}\right),\left(\mathrm{H}_{2}\right)$, and (2.5) are more applicable than that of [7].

(3) We studied the effect of some quadratic terms for (2.4) and obtained many homoclinic orbits and periodic solutions. Also, we explained that the homoclinic orbits make two-dimensional manifolds. Furthermore, we saw that the homoclinic orbits are filled up with the periodic solutions.

(4) By Lemma 2.4 , if $\left(x_{0}, 0\right)$ is a fixed point for $(2.9)$, then $\left(x_{0}+\lambda, 0,0\right)$ is a fixed point for (2.4). Since the periodic solutions obtained by the Hopf bifurcation lie in a neighborhood of $(x(\mu, \lambda), 0)$, so the periodic solutions for (2.4) make a cylinder around the $x$-axis.

\section{REFERENCES}

[1] Z. Afsharnejad, Effect of nonlinear terms $f(x, \dot{x})$ on harmonic oscillator, Iranian J. Sci. Tech. 26 (2002), no. A1, 137-144.

[2] C. Fabry and J. Mawhin, Oscillations of a forced asymmetric oscillator at resonance, Nonlinearity 13 (2000), no. 3, 493-505.

[3] J. Guckenheimer and P. Holmes, Nonlinear Oscillations, Dynamical Systems, and Bifurcations of Vector Fields, Applied Mathematical Sciences, vol. 42, SpringerVerlag, New York, 1990.

[4] J. K. Hale and H. Koçak, Dynamics and Bifurcations, Texts in Applied Mathematics, vol. 3, Springer-Verlag, New York, 1991.

[5] A. C. Lazer and D. E. Leach, Bounded perturbations of forced harmonic oscillators at resonance, Ann. Mat. Pura Appl. (4) 82 (1969), 49-68.

[6] B. Mehri and M. Niksirat, The existence of periodic solutions for the non-linear autonomous O.D.Es, Nonlinear Anal. Forum 5 (2000), 163-171.

[7] - On the existence of periodic solutions for the quasi-linear third-order differential equation, J. Math. Anal. Appl. 261 (2001), no. 1, 159-167. 
[8] S. Wiggins, Introduction to Applied Nonlinear Dynamical Systems and Chaos, Texts in Applied Mathematics, vol. 2, Springer-Verlag, New York, 1990.

O. Rabiei Motlagh: Department of Mathematics, Faculty of Mathematical Sciences, Ferdowsi University, Mashhad, Iran

E-mail address: rabiei@math.um.ac.ir

Z. Afsharnezhad: Department of Mathematics, Faculty of Mathematical Sciences, Ferdowsi University, Mashhad, Iran

E-mail address: afshar@math.um.ac.ir 


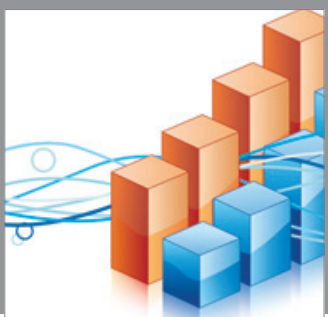

Advances in

Operations Research

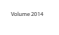

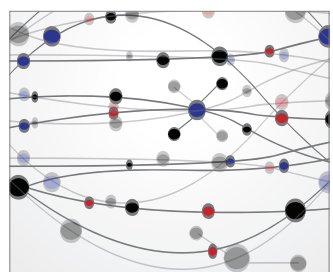

\section{The Scientific} World Journal
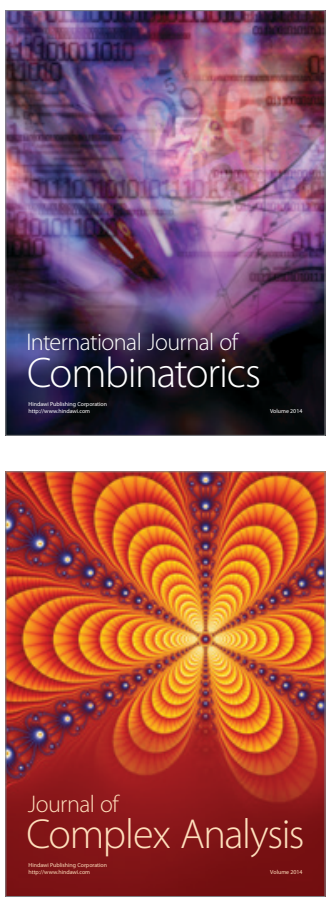

International Journal of

Mathematics and

Mathematical

Sciences
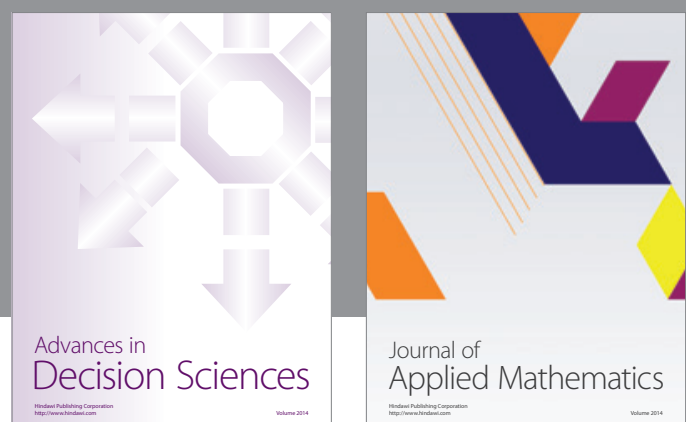

Journal of

Applied Mathematics
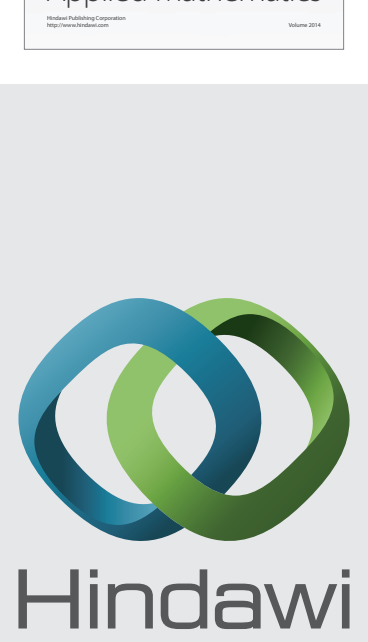

Submit your manuscripts at http://www.hindawi.com
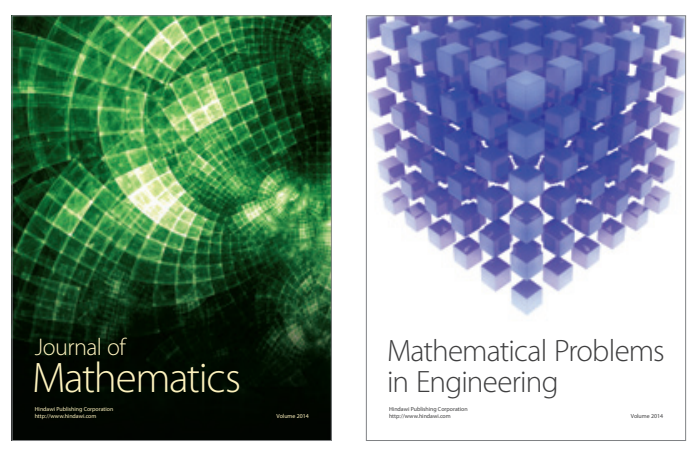

Mathematical Problems in Engineering
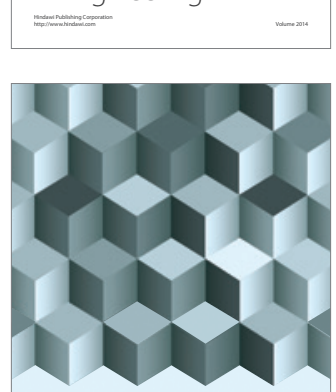

Journal of

Function Spaces
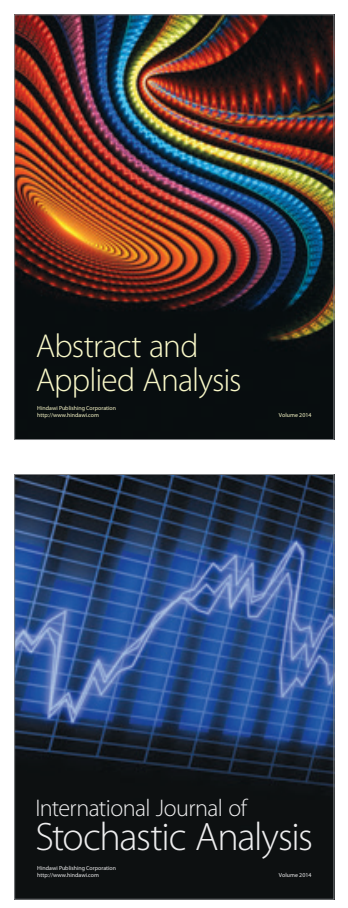

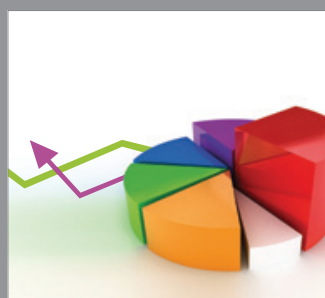

ournal of

Probability and Statistics

Promensencen
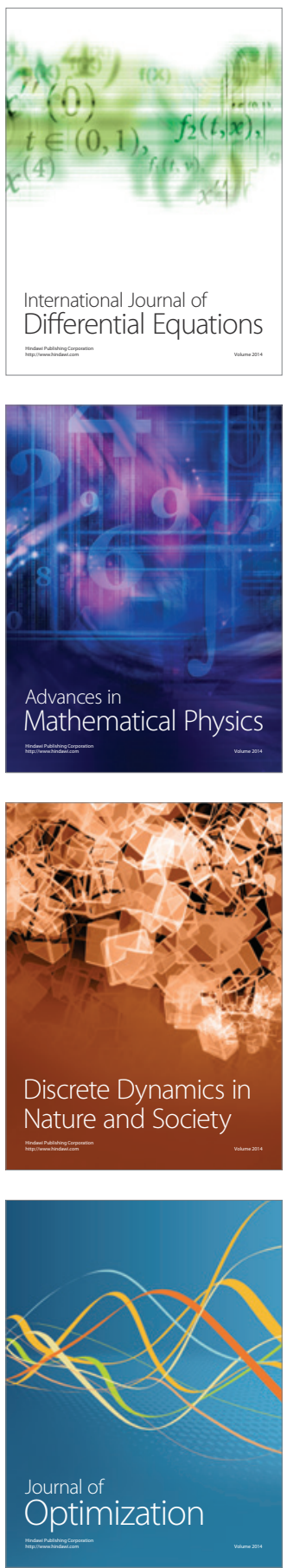